\title{
Problems and Countermeasures for Hospital Ethics Committees
}

\author{
Zijian $\mathrm{Wu}^{1, *}$, Virginia Trigo ${ }^{2}$ \\ ${ }^{1}$ Clinical Research Management Department, Cancer Centre of Guangzhou Medical University,No.78, Hengzhigang \\ Road, Yuexiu District, Guangzhou, Guangdong Province, China 510095 \\ ${ }^{2}$ IscteBusiness School, BRU-IUL, University Institute of Lisbon, Avenida das Forças Armadas, 1649-026, Lisbon, \\ Portugal \\ *Corresponding author. Email: ngken@126.com
}

\begin{abstract}
Hospital ethics committees (HECs) can maximize the protection of the interests and safety of subjects in clinical research, so their functional role cannot be ignored. In China's medical institutions, however, HECs have always been on the edge of management standards and in general have problems such as the lack of organizational structure, unclear management responsibilities and disordered personnel structure, severely curbing the development of ethical review service systems. In the past two years, because of the gene-edited babies incident in 2018 and the rapid development of information technology, the country's social attention in the importance of ethical review is growing widely, which makes medical institutions attach importance to the development of ethics committee. This article takes the $\mathrm{C}$ hospital of my country's third-class $\mathrm{A}$ hospital as an example to introduce the status quo, existing problems and countermeasures of the ethics committee of the hospital in recent years. It is hoped that this article can provide some reference to the development of ethics committees in China's medical institutions.
\end{abstract}

Keywords: ethics committee; review service system; problems; countermeasures

\section{INTRODUCTION}

As medical reform proceeds and society develops, the importance of improving the functions of hospital ethics committees (HECs) and raising the quality of the ethical review system service has become increasingly obvious. This can protect the interests and safety of subjects in clinical research to the greatest extent, and avoid or decrease medical disputes between doctors and patients. Besides, it can also promote the sound development of clinical research in medical institutions by ensuring that scientific research and health care are conducted in accordance with the law and regulations, and increase the influence and recognition of hospital's research results within the country and even across the world. Therefore, it is imperative to further regulate the development of HECs. However, China's medical institutions have not paid enough attention to the development of their ethics committee previously, and most development efforts have not gone beyond top-level designs. One prominent problem is that most medical institutions fail to establish an organizational structure arrange personnel well for ethics committees $[1,2]$. In the internal department management mechanism of public medical institutions in the country, HECs are not included in the management system of traditional administrative institutional organization. And they fail to establish a real departmental for themselves, but exist as an administrative agency under certain departments, such as scientific research and medical management. As a result, the committees' responsibilities are not clearly defined, and the staff have no centralized functions or tight management [3-5]. This article takes Hospital $\mathrm{C}$ as the research subject, and introduces some measures to improve the functions and review service of its ethics committees on the basis of their current situation and existing problems, so as to offer some reference for such committees in China.

\section{BACKGROUND: THE DEVELOPMENT OF HECS GAIN MORE SOCIAL ATTENTION}

\subsection{A storm triggered by a scientific crazy}

HECs have always acted as a "gatekeeper" for clinical research. They plays an important role in protecting the interests and safety of human subjects, and also provide the most important bottom line for researchers and medical institutions in the healthy development of scientific research. The rapid development of modern medical science and technology has benefited patients. But it is also accompanied by potential safety risks, moral deviations and other problems, imposing ever-growing challenges on traditional ethical standards.

At the end of 2018, there was a shocking gene-edited babies incident in Guangdong Province. The incident sparked condemnation from research community, and at 
the same time brought yet another wave of attention and thinking in how to prevent and control wrongdoings in scientific research ethics[6]. China's National Health Commission (NHC), the China Association for Science and Technology (CAST) and other state-level administrative departments have clearly stated: "We will further prompt the management and education of scientific research ethics in research community, and adopt a "zero tolerance" attitude to those who break scientific ethics and norms"[7]. China's healthcare administrative departments at all levels introduced a number of relevant policies and requirements of the development of medical ethics in 2019. For example, HECs are required to implement a record-keeping system; healthcare commissions at all levels should impose dynamic supervision on HECs; the NHC's office of medical ethics expert committee and the Chinese Hospital Association organized experts to study and make the Guide on the Development of Human Research Ethics Review Committees (2019) (hereinafter referred to as the "Guide"); and all clinical researches and technologies for clinical application must receive ethical examination. The "gene-editing" incident pulled the trigger and enabled China's administrative departments at all levels to rethink the importance of the development of ethical norms, and consequently, scientific research standards and ethical and moral inspections are already included in the key development work at the national level.

\subsection{Information technology revolution, such as $5 G$, has spotlighted ethics.}

The new generation of information technology is booming and profoundly changing the way people live and socialize. Now, the information technology revolution represented by Internet Plus, big data, and artificial intelligence is affecting all aspects of human society. Innovations in business processes and technologies are correspondingly made in various industries. As one of frontier disciplines, medicine also bears the imprint of information technology on the development path of the new era. The $5 \mathrm{G}$ technology led by China (now a global leader in mobile network technology) features ultra-high speed, ultra-low latency, and massive connection power and has accelerated the innovation breakthroughs in big data, cloud computing, artificial intelligence and other technologies. These breakthroughs will profoundly affect the entire healthcare industry $[8,9]$. But when the dream of smart healthcare gradually comes true, there are also some potential ethics risks, such as the protection of personal medical data, the definition of the subject-object relation between people and medical products of information technology, and the behaviour standards in telemedicine. All those challenges are confronting medical ethics [10]. This year, with the progress of the "gene editing" incident and the early rollout of 5G technology, all cities, provinces and even the country have attached great importance to the regulation of ethics in science and technology. In January 2019, more than a month after the breakout of the gene-edited baby incident, the government of Guangdong Province issued an official document clarifying the "red line" of scientific and technological innovation and definitely not allowing the bottom line of research integrity and ethics to be crossed [11]. On July 12, 2019, People's Daily, the largest newspaper group in China, published a special editorial entitled Review of Ethics in the Information Age, which discussed the challenges brought by informatization in a full page. On July 24, the ninth meeting of the Central Comprehensively Deepening Reforms Commission of the Communist Party of China made it clear: we should improve the governance mechanism, strengthen supervision in ethics, refine the details of relevant laws and regulations and ethical review rules, and regulate all sorts of scientific research...[12].

From this, we can find that when advancing the development of technology and innovation, China and even the international community have undoubtedly taken some crucial steps to response to the potential risks of science and technology-taking a prudent attitude to early intervene and prevent and control ethical and moral issues that may arise due to the consequences of the application of science and technology and strengthening ethical governance and supervision of science and technology.

\section{THE CURRENT SITUATION OF HOSPITAL C's ETHICS COMMITTEES}

Hospital C, a Class A tertiary hospital affiliated to a university, has established three ethics committees at the same time: medical technology ethics committee, research ethics committee, and Good Clinical Practice (GCP) ethics committee. Their personnel structure and work content vary, and their work is classified as follows: (1) medical technology ethics committee is responsible for the ethical review of medical technology in clinical application, focusing on the reviews of the hospital's new technology and new projects after applying for establishment; (2) research ethics committee for the ethical review of projects in the administrative departments of science and technology at national, provincial, and municipal levels; (3)GCP ethics committee for the ethical review of projects in drug clinical trials and investigator initiated trials (IIT). Over the past two years, with an increasing number of research projects of the hospital, the ethical review and management workload of the ethics committees has become increasingly heavy. Take the GCP ethics committee that is in charge of such review task as an example. Throughout 2019, the GCP ethics committee organized 38 preliminary reviews in GCP projects and 11 in investigator initiated trials, 49 amendment reviews, 28 annual reviews, 85 serious adverse events (SAE), and 58 protocol violations (PV). Compared with 2018, we can find that 2019 saw a sharp rise in the review work (Figure $1)$ 


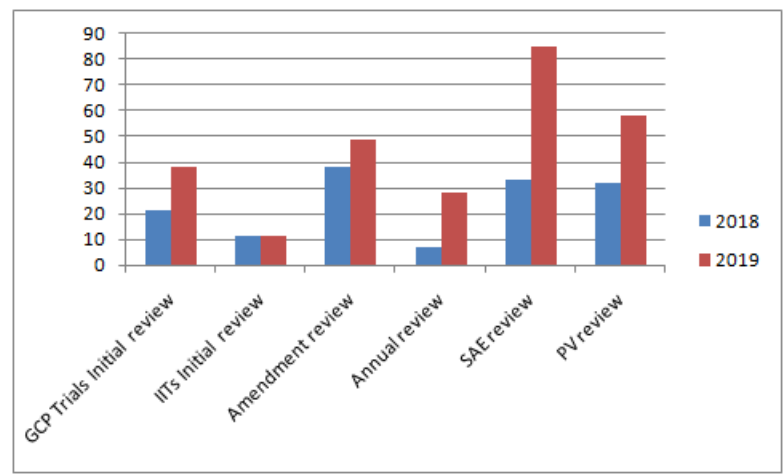

Figure 1 Comparison between GCP ethics committee's review workload of 2018 and of 2019

\section{MAJOR PROBLEMS OF HOSPITAL C's ETHICS COMMITTEES}

\subsection{Problem 1: loose interface management and not unified team, system and procedure}

The ethical review work of Hospital $\mathrm{C}$ is diffused in three different committees which belong to different administrative management lines, and the system construction and SOP implementation of each committee are not consistent (the medical technology ethics committee has not established a systematic management system and SOP; the research ethics committee has only a simple system but without a standardized SOP; the GCP ethics committee's system and SOP are relatively sound). Consequences: (1) Diffused business and blurred boundaries result in unclear responsibility of some projects. As a result, the management personnel of each committee and project researchers would face confusions and troubles in business handling and application, which affects the smooth progress of ethical review. (2) Each ethics committee has differences in the construction of the review service system, operation standards, and work teams. Therefore, the ethical review of Hospital $\mathrm{C}$ finds it difficult to be unified, and the objectivity and accessibility of review results will be questioned and challenged.(3) The systems and SOPs of the medical technology ethics committee and the research ethics committee have not established standards, and the review systems of the three committees are not uniform. A wide gap has thus been created between such system and China's current policies, laws or industry standards, so major inspectors or special reviewers may not give a green light in the future. (4) With inconsistent teams, organizational structure and businesses, the daily management of each committee is hard to conduct and maintain in a systematic way.

\subsection{Problem 2: Unreasonable current personnel structure}

Each Committee's personnel structure lacks certain reasonableness:

\subsubsection{Unreasonable personnel arrangement}

In the Measures for the Ethical Review of Biomedical Research Involving Humans issued by the NHC in 2016 (hereinafter referred to as the "Measures"), “... The members of ethics committee should be selected from experts in the fields of biomedicine, ethics, law, and sociology and other persons beyond the medical institution..." However, the ethics committees' members in Hospital Care all from medical backgrounds and subject to the hospital.

In addition, the Guide requires: "Ethics committees should have one director and several deputy directors...The legal representative of healthcare institution or the head of the scientific research department should not be appointed as director/deputy director." At present, the director of the medical technology ethics committee and the deputy director in the research ethics committee are absent.

\subsubsection{Unqualified personnel}

The Guide mentioned: “... All members should receive basic professional trainings of scientific research ethics and obtain a provincial or higher level relevant certificate before they start their work. Members participating in the ethical review of drug clinical trials should obtain a GCP training certificate recognized by the National Medical Products Administration."A large number of members of the medical technology ethics committee and the research ethics committee have not received standardized training and obtained certificates.

\subsubsection{Organizational structure influence on review meetings}

The Measures and the Guide require: "The effective number of attendees refers that only when members participating in the review should account more than half of the total number of members and include medical persons and non-medical persons, persons independent of the research/trial institution, and people of different genders, the meeting is valid." The basic way the medical technology ethics committee and the research ethics committee review is through simple procedures (review through documents). Considering the requirements of the GCP standards, the GCP ethics committee must conduct a meeting for the preliminary review. With an increasing number of clinical trial projects, it is more and more urgent to organize a review meeting in time to meet the needs of the efficient development of project. However, the number 
of the members outside the hospital in the GCP Ethics Committee is relatively inadequate (only 3, but in principle, at least 2 are required for a valid meeting), so in many cases meetings cannot be not organized as scheduled as two such members are hardly available at the same time.

\section{COUNTERMEASURES}

\subsection{Establish a unified management department}

In March 2020, Hospital C integrated the three original ethics committees into an exclusive, independent and sole ethics committee, and put the existing diffused ethics work under centralized management to eradicate separated work responsibilities and unclear boundaries.

\subsection{Establish new leadership and keep administrative and professional management in parallel}

In 2019, Hospital C conducted a simple research and analysis of the professional background of the directors of ethics committee in 10 hospitals in Guangdong Province. The results revealed that 7 hospitals appointed a hospital' leader as director, and the rest 3 appointed medical professionals. According to the experience of other hospitals, these two situations have both merits and side effects: (1) If the director is a leader of the hospital, ethics committee under his or her leadership can better push work forward, which is beneficial to the implementation and efficiency of the committee's work. With an advantage in administration, the leader can take a strategic view to develop the ethics committee, combined with the hospital's planning goals, thus providing support and a great driver to the development of the committee. However, due to the heavy hospital management work, the leader can only allocate limited energy and time to the management of the ethics committee. (2) Medical professionals, with certain experience and authority in the field, have their own unique, professional influence and judgment on clinical research. But most of them have problems such as inadequate administrative management experience and capacity, so the ethics committee can only receive limited administrative support. Besides, medical experts' inherent academic thinking and one-dimensional sight often keep them away from a comprehensive judgment of clinical research. In the process of ethical review, they focus too much on whether the research is scientific, but ignore the core of ethics and humanity.

Through comprehensive trade-offs, the leadership of Hospital C believes that the hospital leader's integrated management can increase the support for the construction of ethics committee and meanwhile remain independent in review work, which will bring more benefits to the sound development of the committee. Therefore, from March
2020, the critical care medicine expert who is the previous director will serve as the deputy director to take charge of the ethical review of GCP projects; two additional deputy directors who both are medical experts are added to take charge of clinical projects (IIT projects and medical technology projects) and scientific research projects respectively; a deputy CPC committee secretary of the hospital serves as the director of the new ethics committee at the same time. As a result, the ethics committee of Hospital $\mathrm{C}$ has achieved a transition from simple and extensive management led by medical experts to a sound one with a balanced combination of administration and medical profession.

\subsection{Enrich work teams and improve the management level and business quality}

For standardized development, ethics committee must be equipped with a group of members and staff with professional quality, independent spirit and serious attitude. For this, Hospital $\mathrm{C}$ takes the opportunity of the construction of the new ethics committee to further improve the organizational structure and personnel arrangement to build a working team with both professional quality and management ability.

The GCP ethics committee's original members perform relatively well in quality, review experience, and the construction of review system, so most of them make up the majority of the new ethics committee members. And on this basis, because of the needs of ethics work development, another 2 members outside the hospital and 4 members inside the hospital are added. After the newly established ethics committee conducts unified management, the business and work processes of the original three committees need to be retooled and optimized, the work load is growing, and responsibilities are extending. Given such situation, Hospital C established the Ethics Committee Office as an exclusive functional department for the management of the committee. The office is responsible for the formulation of the committee's system and SOP, training, organizational review, document drafting, archives management and other work in an integrated way. The establishment of such office is the basis of and key to standardized ethical review work. And it can better ensure the orderly and high-quality progress of the committee's work, reduce or avoid work mistakes, and better bear the responsibility of protecting the interests and safety of subjects.

\section{OUTLOOK FOR THE CONSTRUCTION OF THE ETHICS COMMITTEE OF HOSPITAL C}

\subsection{Try to obtain the certification of SIDCER}

SIDCER (Strategic Initiative for Developing Capacity in 
Ethical Review) is a special assessment of ethics committee's capacity initiated by the World Health Organization (WHO). It is an international certification project and represents the highest standards of ethical review certification. Its purpose is to develop capacity in ethical review of ethics committees and its goal is to enhance the protection of the interests and safety of human subjects in biomedical and social behaviour researches [13]. Obtaining the SIDCER certification means that the development of a hospital ethics committee has received international recognition. Since early 2020, Hospital C has been actively preparing for the application of that certification, and hopes to further help its ethics committee improve the quality, transparency and social benefits of ethical review through the efforts of striving for the certification.

\subsection{Further improve capacity-building according to laws and regulations}

In April 2020, the National Medical Products Administration issued the latest version of the Good Clinical Practice (hereinafter referred to as the "GCP"). The GCP puts forward new requirements for the ethical review of drug clinical trials and further emphasizes the protection for subjects to their interests and against risks; clarifies that when subjects need medical treatment, they should get timely and standardized diagnosis and treatment in line with diagnostic guidelines, clinical pathways and core systems. While striving for SIDCER certification, Hospital C will implement the new GCP and the Guide requirements, strengthen the capacity-building of its ethics committee, and fully leverage the supervision function of the committee, to improve its ethical review level, and continue to innovate and improve the service system to ensure the safety of subjects and the authenticity and credibility of clinical research.

\section{CONCLUSION}

Under the new situation, China's health administrative departments will strengthen supervision over the normative construction of HECs, which would not only be a challenge, but also an important opportunity to achieve standardized management and improve the professional ability of ethic review. Hospitals should implement the requirements of national policies and regulations, meanwhile, do a good job in the top-level design from the aspects of organizational structure, management system and personnel composition, and strengthen policy support, so as to create a good foundation for the development of HECs. In addition, the HECs also need to enhance the training of professional ability, effectively establish the mission spirit of protecting the rights, interests and safety of the subjects, and fundamentally enrich the connotation and accomplishment. Only when the hardware and software are improved, the review ability of the HECs could achieve a qualitative change and better meet the requirements of the new era.

\section{REFERENCES}

[1]. Yanli, N., et al., Study on Ethical Review of Clinical Research in a Municipal Hospital and Its Countermeasures. medicine and jurisprudence, 2018.

[2]. Minsheng, F.and X, Yiqun, Discussion on the construction of hospital Ethics Committee. Chinese Medical Ethics, 2007(05): p. 9-12.

[3]. Zheng, L. and J. Huang, Development and Administration of Ethics Committee in Hospital Under the New Circumstances. Medical Journal of Wuhan University, 2018.

[4]. Xiaohu, W., et al., Ethical Review Dilemma and Countermeasure Research of Medical Institution Ethics Committee. Journal of Jinzhou Medical University (Social ence Edition), 2019.

[5]. Peili,Z., Yafeng, W.and Y. Chunya, The Function and Construction of Hospital Ethics Committee in China. Medicine and Society, 2010. 23(01):p. 62-64.

[6]. Peng,W., Baby Gene Editing Is a Compliance Wake-up Call for Chinese Research. New Sankei, 2019(03): p. 43-45.

[7]. Yulan,C., Ethical and Social Considerations of "Gene-Edited Babies". Legal Expo,2019. 000(010): p. 29-30.

[8]. Jian,W., A Brief Discussion on the Integrated Development of 5G and Radio and Television Networks. Digital Media Research, 2018(12).

[9]. Zijian, W. and T. Virginia, Impact of information system integration on the healthcare management and medical services. International Journal of Healthcare Management, 2020: p. 1-9.

[10]. Zhonglin,S and Z.Qing., The Relationship Between Subject and Object of Network Public Relations Supervision: Imbalance and Remodeling. China Administrative Reform Forum, 2014

[11]. Jing,Z., Guangdong: Adopt New Measures to Support Innovation-driven Development. Shandong economic Strategy Research, 2019(04): p. 3.

[12]. Xu,L., National Governance in the New Era: Constitutional Practice of Commission for Deepening Overall Reform of the CPC Central Committee.Scientific Socialism, 2019. 000(001): p. 96-103.

[13]. Chen,W, et al.,. Construction and Prospect of Ethics Committee of Drug Clinical Trial. Drug Evaluation, 2011. 08(4):p. 4-8. 